\title{
Executive Functioning in Preschoolers: Reducing the Inhibitory Demands of the Dimensional Change Card Sort Task
}

\author{
David A. C. Rennie and Rebecca Bull \\ School of Psychology \\ University of Aberdeen, Scotland \\ Adele Diamond \\ Center for Developmental Cognitive Neuroscience \\ University of Massachusetts Medical School
}

\begin{abstract}
To investigate the role of inhibitory control in preschoolers' ability to switch sets, 3 conditions of the Dimensional Change Card Sort task ( Zelazo, Reznick, \& Pinon, 1995 ) were tested. In Condition B (novel response options, standard stimuli) action inhibition was reduced, but the need for attentional inhibition was maintained. In Condition C (novel stimuli, standard response options) demands on both action and attentional inhibition were reduced. Performance in these was compared to that in the standard condition (A). Rule complexity was comparable across conditions. All 21 children who passed preswitch (average age 37 months) were tested on all postswitch conditions, order counterbalanced. Although reducing demands on action inhibition (Condition B) did not significantly improve performance, when demands on both action and attentional inhibition were reduced (Condition C) almost all children (95\%) successfully switched sets (even children only $2 \frac{1}{2}$ years old). Inadequate inhibition (of attention alone or both attention and action) appears sufficient to account for virtually all errors by preschoolers on this card sorting task.
\end{abstract}

Executive functioning (EF) is a highly complex set of processes essential to the everyday working of humans when controlled, rather than automatic, processing is required (Shallice, 1988). Although there is debate about what constitutes EF, these executive abilities, considered to be subserved by frontal systems, are evident in tasks such as the A-not-B (Piaget, 1954), Stroop (Stroop, 1935), and Wis-

Requests for reprints should be sent to Rebecca Bull, School of Psychology, William Guild Building, University of Aberdeen, Scotland AB24 2UB. E-mail: r.bull@abdn.ac.uk 
consin Card Sorting (Heaton, Chelune, Talley, Kay, \& Curtiss, 1993) tasks. Important processes subsumed under EF include forming abstract concepts, constructing and using a sequenced plan of action, focusing and sustaining attention or mental effort, manipulating information held in mind, cognitive and behavioral flexibility, being able to inhibit impulsive or inappropriate responses, and being able to self-monitor and detect errors as a task is being performed (Hughes, 2004; Liss et al., 2001). A marked improvement in EF occurs between the ages of 3 and 5 years that is particularly apparent on tasks that require holding information in mind plus inhibition (Diamond, 2002; Diamond \& Taylor, 1996; Gerstadt, Hong, \& Diamond, 1994; Zelazo \& Jacques, 1996; Zelazo, Reznick, \& Pinon, 1995).

Frye, Zelazo, and Palfai (1995) created the Dimensional Change Card Sort (DCCS) task to assess the extent to which young children are able to hold two sets of rules in mind, apply them, and switch between them. The DCCS task requires children to sort picture cards according to shape or color. Initially 3-year-olds have few problems with the task, but many have great difficulty when asked to switch sorting criteria, irrespective of which criterion was used first. From the age of 4 to 5 years and on, most children can successfully switch to sort by the second dimension. It is interesting that children who fail the postswitch phase of the test by sorting perseveratively (continuing to sort by the preswitch criterion) display an apparent dissociation between knowing the rules and using them (Zelazo, Frye, \& Rapus, 1996). For example, 3-year-olds answer knowledge questions ("Can you show me where the red ones/blue ones (stars/trucks) go?") correctly pre- and postswitch, showing that they understand and know the rules. However, during the postswitch they do not apply the rules they have just indicated they know. Instead, they continue sorting by the previously correct dimension. In some ways this is reminiscent of Luria and Homskaya's (1964) observation that adults with frontal lobe damage who consistently verbalized the correct sorting rules, simultaneously sorted cards in such a way as to violate those stated rules. Recent studies examining the performance of children aged 3 to 5 years on other measures requiring holding information in mind plus inhibitory control support a dissociation between children's intact knowledge of rules for the task and their apparent lack of sufficient inhibitory control to correctly apply those rules - for example, go/no-go task (Dowsett \& Livesey, 2000), delay gratification (Mischel \& Mischel, 1983), and the windows task (Russell, Mauthner, Sharpe, \& Tidswell, 1991).

Munakata (2001) and Munakata and Yerys (2001) maintained that this apparent dissociation can be explained in terms of graded knowledge representations, with strength of the representation being dependent on factors such as the stage of development of an individual. Munakata and Yerys suggested that stronger representations are required to resolve conflict, for example, in situations where there might be a conflict between a new rule (color) and an old rule (shape). Answering a standard knowledge question, such as "Where do red things go in the color game?" does not require conflict resolution. However, when children are asked, "Where 
does the red truck go in the color game?" after they have just played the shape game, they err in the verbal response as well as in their manual sorting response (Munakata \& Yerys, 2001), suggesting that there is no dissociation between action and knowledge when the two measures are equated in terms of conflict.

Kirkham, Cruess, and Diamond (2003) argued from the same results that children succeed when memory, but not inhibition, is taxed (nonconflict situations), but fail when inhibition is required (conflict situations), indicating that it is the need for the exercise of inhibitory control that causes problems for the children. Certainly, children have not simply forgotten that the sorting criterion has switched or what it has switched to, as the experimenter emphasizes that rules have been changed and mentions what the new criterion is. Children are reminded of the sorting criterion on every trial, and in some studies are asked to restate the new rules during the task (Zelazo et al., 1995). Children's correct answers to the knowledge questions provide evidence of their intact recall of the rules, Kirkham et al. (2003) argued. Indeed, where children are required to remember more than two rules, but where no switching between sorting criteria is required, children are capable of maintaining the rules in memory and acting correctly (Zelazo \& Frye, 1998).

Another theory attempting to account for children's difficulties on tasks such as the DCCS is the Cognitive Complexity and Control (CCC) theory (Zelazo \& Frye, 1997, 1998). This theory posits a system of levels of rule use, with the level at which a child can function depending on age. According to the CCC theory, 2 -year-olds can only operate at the lowest level. For example, they can correctly sort blue cards into a blue pile, but when given red cards they also put those in the blue pile. At around 3 years of age, children can use a pair of rules; for example, "Blue ones go here and red ones go there" or "Trucks go here and stars go there." It is not until 4 or 5 years of age, according to the CCC theory, however, that children are able to embed both sets of rules in a more complex hierarchical structure; for example, "In the color game, blue ones go here and red ones go there. In the shape game, however, trucks go here and stars go there." That is, if we are playing the shape game, a blue truck should be sorted with the red trucks, not with the blue stars. Thus according to the CCC theory, 3-year-olds do not change their mode of sorting when the sorting criterion changes because they are unable to effectively represent the hierarchical rule structure.

Manipulations of the DCCS task that seem to support the CCC interpretation of why 3-year-olds fail to switch sorting criteria on the DCCS task include the work of Jacques, Zelazo, Kirkham, and Semcesen (1999), who added an error-detection component to the task. By examining children critiquing a third party performing the task, Jacques et al. reasoned that the response control (inhibition) component of the task would be reduced, and thus the ability to apply the rules would be the main ability assessed. Children of 3 years failed the postswitch phase when undertaking it themselves and when critiquing a puppet performing the task. They failed to detect when the puppet sorted perseveratively, and they reported that the puppet 
was wrong when it correctly switched sorting criteria in the postswitch phase. Jacques et al.'s interpretation of this result was that the 3-year-olds' failure on the task occurs because they are unable to represent, manipulate, and reflect on the rules (i.e., their rule-use system is immature). The authors argued that if 3-year-olds' failure on the DCCS task were due to a lack of response control (inhibition), children would have been able to recognize that the puppet was sorting perseveratively. However, this does not preclude the possibility that failure was due to lack of attentional inhibition. Diamond and Kirkham (2001) proposed that a failure of attentional inhibition would be expected to produce the pattern of results obtained by Jacques et al (1999).

Recent findings seem incompatible with the complexity-of-rule-use interpretation. In particular, two recent studies have shown that 3-year-olds can succeed in switching even when they must hold two sets of rules in mind, each set having two subordinate rules apiece, just as is in the DCCS task, as long as the children do not have to switch the dimension to which they are attending (Brooks, Hanauer, Padowska, \& Rosman, 2003; Perner \& Lang, 2002). For example, 3-year-olds can reverse sorting rules, so that in the "sensible" game they sort trucks with trucks and stars with stars and then switch to sorting trucks with stars and stars with trucks in the "silly" game. This hierarchical, embedded rule structure would seem as complex as that in the DCCS task. Presumably there must be some other reason why 3-year-olds succeed here but not on the DCCS task. Indeed, there is considerable evidence (e.g., Roberts et al., 1994; Rogers, Andrews, Grasby, Brooks, \& Robbins, 2000 ) that intradimensional shifts (shifts that stay within a single dimension, as in Brooks et al., 2003, and Perner \& Lang, 2002), are often easier than extradimensional shifts (shifting from one dimension to another, as in the standard DCCS task). The question is, if intra- and extradimensional shifts do not differ in the complexity of rule structure, what is the critical difference that makes intradimensional shifting easier? Often the difficulty in an extradimensional shift is in thinking a bit "outside the box," in realizing that a different dimension might be relevant and in considering what that newly relevant dimension might be. That is not the case with the DCCS task, however, because participants do not have to deduce the relevant dimension, as the relevant dimension is restated by the examiner on every trial.

Diamond and Kirkham (2001; Kirkham et al., 2003) proposed that children fail the standard DCCS task because of "attentional inertia," that is, insufficient inhibitory control of attention to enable 3-year-olds to break their attentional focus on the initial sorting dimension. Contrary to other explanations, Diamond and Kirkham argued that before the stimulus appears, 3-year-olds are ready to perform correctly; they have clearly in mind what the new sorting criterion is, and the appropriate rules for that dimension. This is shown by their correct responses to the knowledge questions. The problem occurs, according to Diamond and Kirkham, when a stimulus card is presented that is relevant to both the pre- and postswitch 
sorting dimensions in incompatible ways (i.e., if sorting by Dimension 1, the card should go here, but if sorting by Dimension 2 the card should go there). Children's attention is pulled to the previously relevant dimension; they must then inhibit that pull. Children of 3 years are thus said to have difficulty redirecting their attention to a newly relevant sorting dimension when the values of the previously correct dimension are still present. They have a problem disengaging from a mindset (a way of thinking about the stimuli) that is no longer relevant, hence showing attentional inertia (see also, Allport, Styles, \& Hsieh, 1994; Allport \& Wylie, 2000). This theory can easily account for the findings of Jacques et al. (1999) because the cognitive set currently held by the children would drive what they expect the puppet to do, that is, to continue sorting by the dimension to which they had previously been attending.

Findings consistent with this point of view include those of Zelazo, Frye, Reznick, Schuster, and Argitis (1995, as cited in Zelazo \& Jacques, 1996). They found that if the stimulus cards were changed only along the dimension that had previously been irrelevant, but maintained the values that had been relevant to sorting during the preswitch phase (e.g., sorting red and blue rabbits by color in the preswitch phase, and blue and red flowers by shape at postswitch), then children still had great difficulty on the postswitch trials and continued sorting according to the preswitch rule (color). Kirkham et al. (2003) argued that the reason for the difficulty is the presence of the previously relevant values of the previously relevant dimension. Zelazo et al. (1995, Expt. 3, cf Zelazo \& Jacques, 1996) found that when the postswitch cards differed along both dimensions (color and shape), younger children were much more successful. Here, rule complexity was unchanged from the standard condition, but the previously relevant values of the preswitch dimension were no longer available to divert children's attention from what they should be focusing on postswitch. This finding would appear to be consistent with the "attentional inertia" hypothesis, but not with CCC theory.

According to Diamond and Kirkham (2001; Kirkham et al., 2003), the familiar target cards, each with a valid value on the previously relevant dimension, serve as attractors, pulling the child to think and act according to the previously relevant rules. Consistent with that idea, 3-year-olds succeed if the previously relevant values on the now irrelevant dimension are no longer present on the target cards or, indeed, when no target cards are present at all (Perner \& Lang, 2002; Towse, Redbond, Houston-Price, \& Cook, 2000). Because that manipulation does not change the complexity of the rule structure of the task, it is hard to see how CCC theory could account for such findings.

Kirkham et al. (2003) administered new conditions of the DCCS task that varied in inhibitory demand. In the "label" condition, for example, instead of the experimenter labeling the relevant sorting dimension of the test card on each trial, the child did so. Kirkham and colleagues reasoned that having children label the relevant dimension of the card to be sorted would help them inhibit attending to the 
previously relevant dimension and help them refocus their attention on the currently relevant dimension. Indeed, significantly more 3-year-olds were able to switch sorting dimensions. In another manipulation, inhibitory demand was increased by having children sort the cards into trays face up, rather than with the picture facing down. After the switch, a child might have to sort a blue truck by color but be faced with seeing a blue truck card under the red truck target card and a red star card under the blue star target card. Hence, the picture sitting in the tray should intensify the perceptual pull to continue sorting by the previous dimension. As predicted, fewer 4-year-olds were able to switch sorting dimensions in the face-up condition than in the standard condition or any other condition.

Another possible reason why 3-year-olds do not switch to sorting by the second dimension on the DCCS task might be that they are unable to inhibit the prepotent response of sorting by the previously correct stimulus-response mappings. Indeed, Towse et al. (2000) argued that the inhibitory failure is at the level of action. If an inability to inhibit a dominant response tendency were the reason 3-year-olds fail to switch on the DCCS task, one might expect that the more times they sorted by the first criterion the more likely they would be to perseverate. However, even after only one preswitch trial, most 3-year-olds fail to switch sorting criteria (Zelazo et al., 1995, as cited in Zelazo \& Jacques, 1996; Zelazo et al., 1996). Their performance after one preswitch trial is not significantly different from their performance after six preswitch trials. That result is not devastating finding for this perspective, however, because a bias or conditioned tendency can be instilled by just one trial (e.g., Thompson, 1990). However, Jacques et al.'s (1999) findings would also be inconsistent with a response-inhibition interpretation.

Inhibition of action and inhibition of attention might reflect different processes, even though both involve inhibition. For example, Mayr and Keele (2000) proposed that there are two types of inhibitory processes involved in switching tasks. One, the need to resist goal-incongruent action tendencies (stimulus-response mappings), and two, the need to flexibly change attentional control settings. Bunge, Dudukovic, Thomason, Vaidya, and Gabrieli (2002) reported different patterns of maturation, and different patterns of neural activation, for interference suppression (inhibition of attention) and response inhibition (inhibition of action).

Our study looked at the effect of reducing the demand on inhibition of action, and of reducing the demand on inhibition of both action and attention, on preschoolers' performance of the DCCS task. The three conditions of the DCCS task varied in the level of inhibition required but maintained the same level of rule complexity (two rule sets, color and shape, each with two subordinate rules), and all used cards with stimuli that had both color and shape. In the standard DCCS condition (Condition A), blue trucks and red stars served as the stimuli, and a blue star and red truck were each pictured on one of the target cards. Each target card was mounted over one of the two sorting bins (see Figure 1). 
Correct responses when sorting by COLOR:

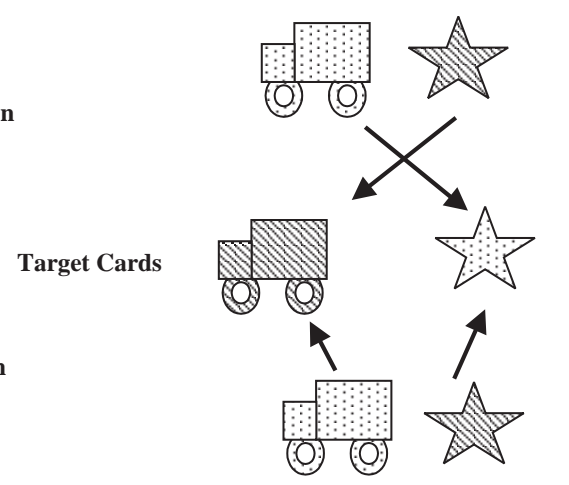

\section{Correct responses when sorting by COLOR:}

Correct responses when sorting by SHAPE:

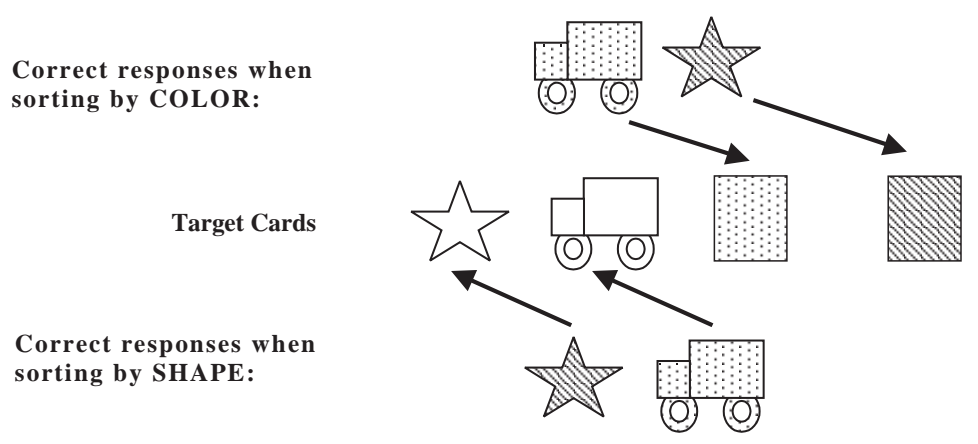

\section{Correct responses when sorting by COLOR:}

\section{Target Cards}

Correct responses when sorting by SHAPE:

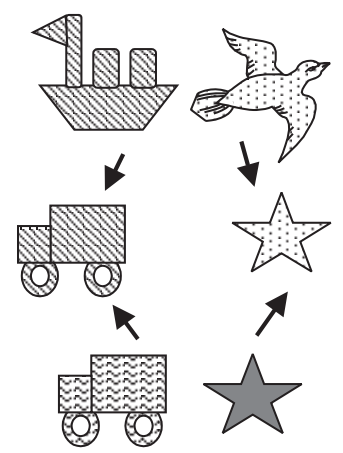

FIGURE 1 Illustration of the structure of the three DCCS conditions tested here. Arrows show correct sorts. The top panel illustrates Condition A: Standard DCCS. The middle panel illustrates Condition B: Reduced Action Inhibition (univalent target cards). The bottom panel illustrates Condition C: Reduced Attentional and Action Inhibition (each stimulus card matched only one target card and along only one dimension; different stimulus cards used pre-and postswitch). 
In Condition B, the response options were changed. Four sorting bins were used, and the target card mounted over each was unidimensional (i.e., "univalent:" colorless truck or star against a white background, or red or blue rectangle). The same stimulus cards as in Condition A were used. Because the stimulus cards still contained relevant values along both dimensions, if attention were not inhibited to the wrong dimension, a response option was available to sort by that wrong dimension. The advantage of Condition $\mathrm{B}$ over the standard condition is that the correct answers here for the postswitch trials were not answers that had been relevant (actively inhibited) during the preswitch phase. In Condition B, children still had to understand and remember which dimension was currently relevant, and they still had to switch the focus of their attention from one dimension to another, but different stimulus-response mappings were relevant pre- and postswitch so that the old stimulus-response mappings did not have to be overridden. Therefore, this condition decreased the possibility of perseveration at the level of action (see Figure 1).

In Condition C, the same response options (same target cards over the same two bins) as in the standard condition were used, but the stimuli were changed. The stimulus cards used here were yellow and green trucks and stars for sorting by shape, and red and blue birds and boats for sorting by color. Hence, any given stimulus card matched only one target card and did so along only one dimension; no inhibition was required because only one response option was relevant to any given stimulus card. Here, old stimulus-response mappings did not need to be inhibited (they were not an option postswitch), and attention to the previously relevant dimension did not need to be inhibited (no relevant values on the previously relevant dimension were presented during the postswitch phase). Hence, this condition reduced the necessity for inhibition at the level of attention and action (see Figure 1).

Our predictions were that (a) children of 3 years would fail the standard task (Condition A), that is, they would not switch sorting dimensions; (b) more 3-year-olds would succeed on Condition B (reduced demand on action inhibition) than Condition A, and a greater number of the younger children would succeed as well; (c) Condition C (reduced demand on attentional and action inhibition) should be the easiest condition; most 3-year-olds would pass (i.e., successfully switch sorting dimensions).

\section{METHOD}

\section{Participants}

Thirty-three children between the ages of 30 and 46 months ( $M=37$ months, $S D=$ 4 months) were tested. There were 17 girls and 16 boys. All were British White children, with English as their first language, recruited through private day care centers located in lower middle-class areas in Aberdeen, Scotland. Children were 
tested following informed parental consent. Children with known developmental delays (as identified by the teacher) did not take part in the study. All children were tested in all DCCS conditions, with the order of conditions and initial stimulus dimension to be sorted (shape or color) counterbalanced within each gender and among the oldest half and youngest half of the children.

\section{Materials}

Laminated $15-\mathrm{cm} \times 12-\mathrm{cm}$ test cards were sorted into $30-\mathrm{cm} \times 21-\mathrm{cm} \times 10-\mathrm{cm}$ boxes. The height of the back of the boxes was raised to $24 \mathrm{~cm}$ to accommodate the laminated target cards, which were situated $12 \mathrm{~cm}$ above the level of the box and were always at the child's eye level during testing. On each of the cards used, the picture was situated in the middle of the card with a white background behind the picture and a black border on the edge of the card, except for the single-color cards in Condition B, which were the same size, but the entire card was of the color required. Target cards for Condition A were a blue star and a red truck, and the stimulus cards were a red star and a blue truck. Training cards were blue and red grapes and yellow trucks and stars. In Condition B, the four target cards were just the outline drawings of a truck or star (i.e., no color fill) against a white background, and entirely blue or entirely red cards. The stimulus cards and training cards were the same as in Condition A. The target cards in Condition C were the same as Condition A. The stimulus cards for Condition $\mathrm{C}$ were yellow trucks and green stars and red boats and blue birds. The training cards were red and blue grapes and colorless trucks and stars. Colorless rather than yellow training cards were used because the stimulus cards were colored yellow and introducing a new color into the task might have been confusing. The cards matched exactly those used by Kirkham et al. (2003) with the addition of colorless stimulus cards and cards that were entirely red or blue.

\section{Design}

The study incorporates a within-subjects design with three levels (standard, reduced response inhibition, and reduced attentional and response-inhibition DCCS conditions). There were four between-subject factors: condition order, dimension order (sorting by shape or color first), gender, and age. Children were randomly assigned to the order in which the conditions and the stimulus dimensions were presented. The dependent variable was the accuracy in sorting the card on each trial. Children were required to achieve five consecutively correct responses out of a total of six trials to pass the preswitch condition, and five consecutively correct responses out of six trials to pass the postswitch. 


\section{Procedure}

\section{Procedures Relevant to All Conditions}

Children were asked to sort stimulus cards one at a time into sorting trays according to one of two criteria, color or shape, and then by the other criterion. Stimulus cards were always presented to the children face up and in the middle, between the target trays (between the two trays in Conditions $\mathrm{A}$ and $\mathrm{C}$ and between Trays 2 and 3 in Condition B). The children were given a short introduction to the task, followed by training on each dimension with stimulus cards that matched the target cards on only one dimension (for the color game the training cards had pictures of blue and red grapes, and for the shape game the training cards had pictures of the relevant shapes, trucks and stars, in a neutral color, yellow). Training took place once the child was comfortable and settled, with the experimenter giving the following instructions:

Now we are going to play a card game. In this game we play the color (shape) or shape (color) game. In the color (shape) game all the blue ones (trucks) go in this tray (experimenter points), and all the red ones (stars) go in this tray (again, experimenter points).

These sentences were used again later in the session when the rules were restated between trials. Next, the child was asked, "Can you point and show me where the blue/red ones (trucks/stars) go?" (Child points, experimenter praises if correct, and restates the rules if incorrect.) "Here's a blue one (truck). Where does it go?" (Child sorts card by placing it face down in a tray. Experimenter praises if correct, and corrects the child if wrong.) "Here's a red one (star). Where does it go?" To pass the training and continue with the game, the child had to correctly sort four cards (two for each dimension) and was only allowed a total of eight attempts during training. All children passed the training phase.

Test trials began immediately after the last training trial with the experimenter saying, "Now we are going to keep playing the shape (color) game" and was based on the dimension last sorted (e.g., if the last training card was a yellow truck, the first test dimension would be shape). Half the children started with color first and half with shape; this was true for each of the three conditions (A, $\mathrm{B}$, and $\mathrm{C})$. When each test card was shown, the experimenter said, "Here is a truck (star, red one, blue one). Where does it go?"-always labeling the card along the currently relevant dimension. The cards were presented in a predetermined pseudorandom order.

After the trials at the first dimension (preswitch), the game was switched to the other dimension. This postswitch phase was initiated by the experimenter saying, "Now we are going to play the other game. Do you remember the color (shape) game?" The experimenter then restated the rules and asked knowledge questions 
to make sure the child knew how to sort the cards correctly according to the new dimension. All children answered the knowledge questions correctly. Testing on the second dimension continued until the child achieved five consecutive correct responses out of the six postswitch trials administered. During either phase (pre- or postswitch), if the child sorted incorrectly five times in a row, the experimenter reverted back to the training cards for the dimension currently in use and administered those cards until the child sorted both training cards correctly. After this, the experimenter continued with more postswitch trials. At the end of the testing each child was praised for his or her performance.

During the intertrial intervals, the experimenter alternated between restating the relevant rules or asking the child knowledge questions for each of the two currently relevant rules ("Can you tell me where the red ones/blue ones (stars/trucks) go?"). No feedback was given throughout the test trials on the sorting of any card. Feedback was only given to responses to knowledge questions between trials. Sorting by the first dimension continued until the child made five consecutive correct responses, or until the first six trials had been administered. Children who failed to pass the preswitch trials continued with the postswitch phase, although their data was not included in the postswitch analysis $(n=12)$ because preswitch failure indicated inadequate establishment of the prepotent response set. All children who passed preswitch also answered all knowledge questions correctly.

\section{Condition Specific Procedures}

Condition $A$. This is the baseline condition, comparable to the original card sort procedure of Zelazo et al. (1996) and identical to that of Kirkham et al. (2003), described previously.

Condition $B$. This is the univalent response or reduced action-inhibition condition, where the relevant response options changed from pre- to postswitch. Here, the inhibition that had been instituted during the preswitch ("Don't go to red, go to blue on this trial" or "Don't go to the truck, go to the star this time") did not have to be reversed. In this condition there were four trays on the table throughout training and testing, with univalent target cards (colorless star, colorless truck, blue card, red card) attached to them (see Figure 1). The trays faced the child in this order: star, truck, blue, red. In all other respects the procedure here was identical to that used in Condition A.

Condition C. This is the reduced attentional and action-inhibition condition. Although each stimulus still had a color and a shape, any stimulus card only matched the target cards along one dimension. The stimulus cards were yellow trucks and green stars and red boats and blue birds. The training cards were red and blue grapes and colorless trucks and stars. The response trays and target cards were 
the same as in Condition A. Thus, for example, a green truck matched no target card by color, but matched the red truck target card by shape (see Figure 1). Therefore, this condition decreased the need for inhibition of an irrelevant dimension and did not require that stimulus-response mappings be changed. The procedure was the same as in Condition A except (a) the stimulus cards matched the target cards along only one dimension, and (b) the shape dimension was trained by using colorless trucks and stars and not trucks and stars in a neutral color.

\section{RESULTS}

Initial analyses examined the number of children correctly sorting five or more cards on preswitch trials for each condition to investigate whether there were any differences in performance across the different DCCS conditions prior to the category switch. This analysis also investigated whether any of the confounding variables (age, gender, condition order, and dimension presentation order [color vs. switch first]) had an effect on performance. Generalized estimating equations (GEEs) were carried out on the data to take account of the repeated measures structure of the data (see Table 1 for a summary of the results). The GEE model used an exchangeable correlation structure, binomial error and logit link.

This analysis showed that there was a marginal, nonsignificant difference in the number of children passing the preswitch phase across the three DCCS conditions $(Z=4.94, p=.09)$. A total of 12 children failed the preswitch phase (1 child failed Conditions A and B, 7 failed Condition A only, 2 failed Condition B

TABLE 1

Generalized Estimating Equations Analysis of Dimensional Change Card Sort Task Pre- and Post

Switch Performance as a Function of Potential Confounding Variables

\begin{tabular}{lcc}
\hline Variable & Z Value & $p$ Value \\
\hline Pre switch $(N=33)$ & & \\
$\quad$ Age & 0.18 & 0.85 \\
Gender & 0.19 & 0.85 \\
Condition order & 5.93 & 0.05 \\
$\quad$ Dimension order & 0.11 & 0.91 \\
Post switch $(N=21)$ & & \\
Age & 1.08 & 0.28 \\
Gender & 1.10 & 0.27 \\
Condition order & 1.21 & 0.55 \\
Dimension order & 0.54 & 0.59 \\
\hline
\end{tabular}

Note. $\mathrm{GEE}=$ Generalized Estimating Equations; $\mathrm{DCCS}=$ Dimensional Change Card Sort Task 
only, and 2 failed Condition C only). Furthermore, the order in which the conditions were presented was related to the likelihood of passing each of the preswitch conditions $(Z=5.93, p=.05)$. Children were significantly more likely to pass the preswitch trials of Condition A if they had completed that condition first, rather than when it followed either of the remaining DCCS conditions. Thus, experience with the easier conditions appeared to have been helpful to children when subsequently tested on the standard DCCS condition. The preswitch trials for Condition A were slightly more complex (because of the bivalent target and response cards) than those for Conditions B and C. However, in all conditions, the stimulus cards were only labeled according to the currently relevant dimension. Children who failed a preswitch phase of any condition were removed from all postswitch analyses, leaving a sample of 21 children. The sex ratio and mean age of the remaining children did not differ from that of the total sample (50\% girls, $M$ age $=37$ months, $S D=4$ months).

To determine the most appropriate method for analyzing performance on the postswitch trials, the frequency and distribution of the number of correct responses in each condition were examined. Figure 2 shows that in the majority of cases, children's performance was consistent throughout postswitch (i.e., six correct sorts or no correct sorts). Very few children showed success on some trials and not others. Therefore, it is appropriate to treat success-failure as a dichotomous variable. Again, GEE analysis was conducted to check for any confounding effects in order of presentation of task conditions, order of sorting by stimulus dimension, age, or gender on success outcome in the three DCCS conditions. Children were deemed to have passed if they made at least five consecutively correct sorts within

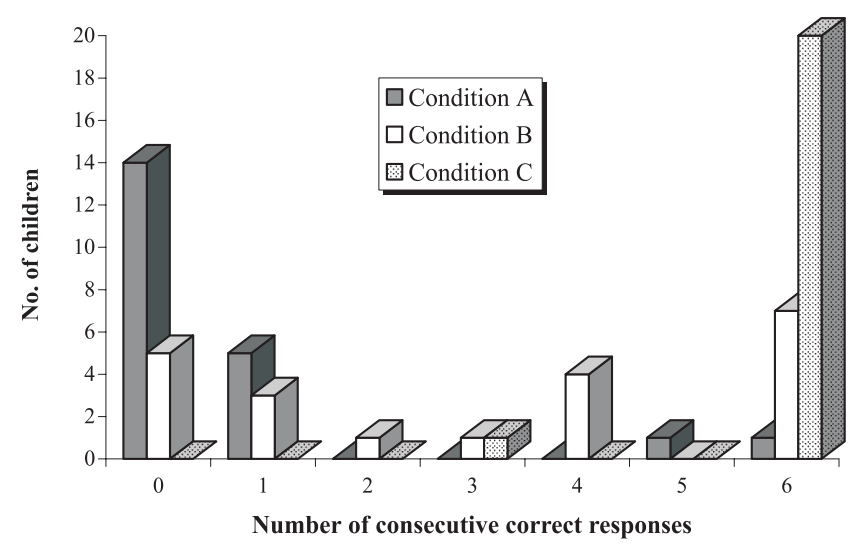

FIGURE 2 Number of children showing $0,1,2,3,4,5$, or 6 correct consecutive postswitch responses in Condition A (standard), Condition B (reduced action inhibition), and Condition C (reduced attentional and action inhibition). 
six postswitch trials. None of the potentially confounding variables (or interactions among the variables) were found to have a significant effect on the likelihood of passing or failing the postswitch trials (see Table 1).

Observation of the raw data in Figure 2 shows that the number of children passing in each of the postswitch conditions differs dramatically, with only 2 children (10\%) passing the postswitch in Condition A, $7(33 \%)$ passing in Condition B, and 20 (95\%) passing in Condition C. Due to the very low failure rate in Condition C and the very high rate of failure in Condition $\mathrm{A}$, it was not possible to estimate the effect of condition using GEE to account for the repeat observations per child. However, a lower order Cochran's $Q$ analysis was conducted, a test that is particularly suitable when the data are in a nominal scale or are dichotomized ordinal information (Siegel, 1956). This analysis revealed a significant difference in performance in the three postswitch conditions $Q(d f=2, N=21)=25.52, p<.001$. Analysis of each pairwise comparison using the McNemar Test revealed that children were significantly more likely to pass Condition $\mathrm{C}$ than Conditions $\mathrm{A}$ and $\mathrm{B}$ (all $p \mathrm{~s}<.001$ : C vs. A, C vs. B, and C vs. A \& B), but there was no significant difference in performance between Conditions $\mathrm{A}$ and $\mathrm{B}(p=.18) .{ }^{1}$ The increase in numbers of children successfully switching dimensions in Condition $C$ was not restricted to just the oldest children in the sample, but was seen even in children only 30 months old.

\section{DISCUSSION}

The failure rate for children around age 3 years on the postswitch phase of Condition A (standard DCCS procedure) was very high, indeed higher than predicted. It was expected that a majority would fail, as most children cannot switch dimensions on the standard DCCS test until about 48 to 60 months of age (Kirkham et al., 2003; Zelazo \& Jacques, 1996; Zelazo et al., 1995), and the average age of children in this study was only 37 months. The higher than typical failure rate may have been due to the very young age of our participants. Reducing the need for action inhibition (Condition B) resulted in more children passing the task, but not significantly more than in the standard DCCS condition. When demands on both attentional and action inhibition were reduced (Condition C), significantly more

\footnotetext{
${ }^{1}$ Pairwise comparisons were conducted both (a) excluding all subjects who failed the preswitch phase in any of the three conditions and (b) only excluding those subjects who failed the preswitch phase of either of the two relevant conditions for each pairwise comparison. In all cases, performance in Condition $\mathrm{C}$ was significantly better at the $p<.001$ level than performance in Condition A or B. When only those participants who failed the preswitch phases of Conditions A or B were excluded from that pairwise comparison, the significance level improved from $p<.18$ to $p<.11$, but it still failed to reach the threshold for being considered statistical significant.
} 
children succeeded at the postswitch phase than in Conditions A or B, with $95 \%$ of children successfully switching sorting dimensions, including children as young as $2 \frac{1}{2}$ years of age.

Before discussing the results for each condition in more detail, a few cautionary notes are necessary. First, the number of participants (21) who correctly sorted according to the initial sorting dimension in the preswitch phase was relatively small, and hence the number of participants who could be included in analyses of postswitch performance was comparably small. However, that sample size of 21 is larger than the number of participants tested in each condition in other DCCS studies - in some cases over twice as large. Furthermore, limited information regarding sociodemographics and other cognitive skills of the participants limits the generalizations that can be made to the population as a whole.

\section{Performance on Condition A}

In the standard condition of the DCCS task there is (a) a need to inhibit the stimulus-response mappings relevant preswitch (action inhibition) and replace them with opposing stimulus-response mappings postswitch and (b) a need to stop focusing on the properties of the dimension that had been relevant preswitch (attentional inhibition), instead focusing on the previously ignored dimension. Virtually all children failed to switch sorting dimensions in Condition A, which required inhibition at both the level of attention and action. This result is consistent with the myriad of previous findings using the standard DCCS task (e.g., Zelazo et al., 1996), although the failure rate here $(90 \%)$ is particularly high. That might be due to the unusually young age of participants in this study (many not yet 3 years old). It is not until 4 or 5 years of age that children become skilled at extradimensional set shifting, even when no hypothesis testing or deduction is required to figure out which dimension is newly relevant.

\section{Performance on Condition B}

Condition B presented four univalent response options, each relevant to only one dimension (e.g., a colorless truck). Children could err by responding to the previously relevant sorting dimension, as each stimulus card matched one target card by shape and another by color. Because the stimulus cards still contained relevant values along both dimensions, children could focus on the wrong dimension if attention were not inhibited. More important, however, correct answers postswitch were not answers that had been relevant preswitch (thus they had not been actively inhibited or "negatively primed"). Also, because different responses (different stimulus-response mappings) were relevant pre- and postswitch, the need for action inhibition was greatly reduced. If children fail because of a difficulty inhibiting an action tendency, as opposed to difficulty refocusing attention onto the rele- 
vant dimension, then 3-year-old children should be more successful in this condition. The findings show that this was not the case, although the success rate was somewhat higher in this condition. It may be the case that because the locus of attention on the preswitch trials was spatially distinct from the postswitch trials (i.e., different sorting bins were used on the postswitch trials), the tendency to see the previous dimension as relevant may have been diminished. Therefore, we were not able to rule out the possibility that in some way, attentional inhibition (even if only to a small extent) was also reduced in this condition, accounting for the increase (although nonsignificant) in success rate.

Towse et al. (2000) also compared two-tray and four-tray conditions. Unlike here, they found most children succeeding when four sorting trays were used. However, they also found most children succeeding in the standard two-tray DCCS condition, making interpretation of their results difficult. The children in Towse et al.'s study were significant older (approximately $42-52$ months of age) than the children tested here, which may account for why they performed so well in conditions resembling our Conditions A and B.

\section{Performance on Condition C}

Condition $\mathrm{C}$ reduced demands on both the attentional and action inhibition required for switching. Attentional inhibition was reduced because each stimulus card only matched one target card, and along only one valid dimension. For example, because the target cards were a blue truck and a red star, if the preswitch sorting dimension was color, children were shown stimulus cards with blue and red birds and boats. Both shape and color dimensions were shown, but only the color dimension matched any target card. At postswitch (shape), the child was presented with green and yellow trucks and stars. Now, the presented values on the previously correct dimension (color) were no longer valid. They did not match the color of any target card. The rule structure was the same as for Conditions A and B, however (two if, then rules for color and two if, then rules for shape).

In the terminology of Diamond and Kirkham (2001; Kirkham et al., 2003), there was no "attentional inertia" in Condition C. Their theory stated that depending on which criterion was used first, the child may become fixed on the "blueness" or the "truckness" of the stimuli and may have trouble inhibiting attention to that dimension. Because the values along the preswitch dimension changed (and were not valid-i.e., matched no target card) at postswitch, children should not have had difficulty inhibiting their focus on, for example, "blueness," as "blueness" at postswitch was no longer present. No postswitch stimulus card contained blue. Hence the switch to attending to the relevant dimension (e.g., "truckness") should have been easier.

Also, no remapping of stimulus-response associations was needed here. It was not the case that during preswitch one response was correct for a red bird stimulus 
and during postswitch another response was correct for that stimulus. The red bird stimulus was never presented during the postswitch phase. Because there was no overlap of stimulus cards pre- and postswitch, no response to any stimulus card needed to be remapped from the pre- to the postswitch phase.

By reducing the demand on attentional and action inhibition, more children could successfully make the switch from sorting by shape to color (or vice versa), with $95 \%$ of children passing the postswitch trials despite their young age. We are not able to conclude from these results whether such a high rate of success in this condition resulted from the reduction in attentional inhibition, or from the conjunction of reduced action and attentional inhibition. Clearly, the answer to this would be found by using a condition that only reduced attentional inhibition. Kirkham et al. (2003) constructed a condition that required switching the stimulus-response mappings (i.e., the action inhibition) but which made the correct sorting dimension at postswitch more salient by having the children label the stimulus card (see also, Towse et al., 2000). It was proposed that this modification reduced the attentional inertia to the previously correct dimension, and it did indeed result in better sorting performance. What can be concluded from these results is that inhibitory demands on attention alone, or the conjunction of attention plus action (although not action alone), can account for essentially all of the difficulty that 3 -year-olds, and even 21/2-year-olds have on the DCCS task. By reducing inhibitory demands in Condition C, virtually all the children were able to succeed.

\section{A LARGER CONTEXT IN WHICH TO CONSIDER OUR RESULTS}

Improvement in performance, produced by reducing demands on attentional inhibition, or attentional plus action inhibition, is in line with results with other cognitive tasks where demands on attentional inhibition were minimized by reducing the perceptual salience of the stimulus dimensions. For example, in an appearance-reality task, the perceptual salience of the stimulus was decreased by removing the object from view during questioning, resulting in significantly better performance by 3- or 4-year-old children (Herbele, Clune, \& Kelly, 1999). Children of 3 or 4 years generally fail tests of liquid conservation (they tend to focus exclusively on the most perceptually salient of the two dimensions; Flavell, 1963). If the salience of the visual perception that the tall, thin container contains more liquid is mitigated by placing an opaque screen between the child and the containers before the child answers, children 3 to 4 years of age can succeed (Bruner, 1964). Similarly, manipulations that reduce the perceptual salience of the true state of affairs on false belief and theory of mind tasks enable children of 3 or 4 years to succeed (such as telling the children where the object is really hidden but never actually showing them; Zaitchik, 1991). 
Reducing the inhibitory demands required for switching sorting criteria may be accomplished by removing the target cards (Perner \& Lang, 2002; Towse et al., 2000), having children label the stimulus card themselves to refocus attention on the relevant dimension (Kirkham et al., 2003), or by eliminating the need to refocus attention by making values of the previously relevant stimulus dimension invalid (our study; Zelazo et al., 1995). In all of those conditions, children of 3 years are able to shift set and succeed at the task. Conversely, studies have shown that increasing demands on attentional inhibition can cause children of 3 or 4 years, who would otherwise succeed, to fail. Inhibitory demands imposed when shifting set are much less when the switch is intradimensional rather than extradimensional (as the former requires no attentional inhibition to a previously relevant dimension); 3-year-olds succeed at the former but not the latter (Brooks et al., 2003; Perner \& Lang, 2002). However, Brooks et al. (2003) found that when a second dimension (color) was added to black-and-white line drawings that indicated shape, 3 -year-olds then failed the reversal task, although color was never used or referred to in the experiment; it was simply present. Hanauer and Brooks (2003) found age-related improvements in children's ability to selectively attend to a visual Stroop task and ignore conflicting auditory cues (for example, hearing the word purple when green is the answer). They could correctly identify the colors when they were presented without conflicting stimuli, but they were unable to selectively attend to the visual stimuli when required to inhibit attention to auditory stimuli.

Most studies of attentional and action inhibition have begun testing at 3, 31/2, or 4 years. It is interesting that in this study we found no difference in performance between $2 \frac{1}{2}$ and $3 \frac{1}{2}$ years. Children of 36 to 46 months performed as poorly as children of 30 to 35 months when inhibition was required, and children of 30 to 35 months performed as well as children of 36 to 46 months when demands on inhibition were reduced.

In summary, decreasing the inhibitory demands of the DCCS task enabled younger children to switch from a color task to a shape task or vice versa. The results of Condition $\mathrm{C}$, in which inhibition was reduced at both the levels of attention and action, indicate that inadequate inhibitory control can account for a large proportion of the difficulty that children of 3 years have with the DCCS task. All 3 -year-olds (indeed almost all 21/2-year-olds) were able to succeed in making the switch when inhibitory control was not required (Condition C). With no inhibitory control required, this task essentially becomes a measure of the ability to hold rules in memory and to represent a hierarchical, embedded rule structure. Clearly, the evidence from this study, along with previous research findings, supports the idea that when the external environment is structured in such a manner as to reduce the inhibitory demands, children of $2^{1 / 2}$ to 3 years have the memory and representational skills to be able to learn, remember, and flexibly deploy rule sets. 


\section{ACKNOWLEDGMENTS}

This research was completed in partial fulfillment for the BSc degree of David A. C. Rennie (University of Aberdeen, Scotland).

Sincere thanks go to all the staff and children from the participating nurseries; and to the staff at the Center for Developmental Cognitive Neuroscience at the Shriver Center, UMass Medical School, for their training of the tester, help with the preparation of the testing materials, and their support and inspiration, which contributed greatly to the production of this research. Thanks also to three anonymous reviewers for their insightful comments on a previous version of this manuscript.

\section{REFERENCES}

Allport, A., Styles, E. A., \& Hsieh, S. (1994). Shifting intentional set: Exploring the dynamic control of tasks. In C. Umilta \& M. Moscovitch (Eds.), Attention and performance XV (pp. 421-452). Cambridge, MA: MIT Press.

Allport, A., \& Wylie, G. (2000). Task switching, stimulus-response bindings, and negative priming. In S. Monsell \& J. Driver (Eds.), Control of cognitive processes: Attention and performance XVII (pp. 35-70). Cambridge, MA: MIT Press.

Brooks, P. J., Hanauer, J. B., Padowska, B., \& Rosman, H. (2003). The role of selective attention in preschoolers' rule use in a novel dimensional card sort. Cognitive Development, 18, 195-215.

Bruner, J. S. (1964). The course of cognitive growth. American Psychologist, 19, 1-15.

Bunge, S. A., Dudukovic, N. M., Thomason, M. E., Vaidya, C. J., \& Gabrieli, J. D. E. (2002). Immature frontal lobe contributions to cognitive control in children: Evidence from fMRI. Neuron, 33, 301-311.

Diamond, A. (2002). Normal development of prefrontal cortex from birth to young adulthood: Cognitive functions, anatomy, and biochemistry. In D. T. Stuss \& R. T. Knight (Eds.), Principles of frontal lobe function (pp. 466-503). London, England: Oxford University Press.

Diamond, A., \& Kirkham, N. (2001, October). Card sorting by children of 3 \& 4 years and task switching by older children: Inhibition needed to overcome "attentional inertia." In Rule use through the lens of the Dimensional Change Card Sort: What develops? Symposium conducted at the meeting of the Cognitive Development Society, Virginia Beach, VA.

Diamond, A., \& Taylor, C. (1996). Development of an aspect of executive control: Development of the abilities to remember what I said and to "Do as I say, not as I do." Developmental Psychobiology, 29, 315-334.

Dowsett, S. M., \& Livesey, D. J. (2000). The development of inhibitory control in preschool children: Effects of "executive skills" training. Developmental Psychobiology, 36, 161-174.

Flavell, J. H. (1963). The developmental psychology of Jean Piaget. Princeton, NJ: Van Nostrand.

Frye, D., Zelazo, P. D., \& Palfai, T. (1995). Theory of mind and rule-based reasoning. Cognitive Development, 10, 483-527.

Gerstadt, C. L., Hong, Y. J., \& Diamond, A. (1994). The relationship between cognition and action: Performance of children 3.5-7 years on a Stroop-like day-night test. Cognition, 53, 129-153.

Hanauer, J. B., \& Brooks, P. J. (2003). Developmental change in the cross-modal Stroop effect. Perception and Psychophysics, 65, 359-366. 
Heaton, R. K., Chelune, G. J., Talley, J. L., Kay, G. G., \& Curtiss, G. (1993). Wisconsin Card Sorting Test Manual: Revised and Expanded. New York: Psychological Assessment Resources.

Herbele, J., Clune, M., \& Kelly, K. (1999, April). Development of young children's understanding of the appearance reality distinction. Paper presented at the biennial meeting of the Society for Research in Child Development, Albuquerque, NM.

Hughes, C. (in press). The development of executive functions. In B. Hopkins (Ed.), Cambridge encyclopedia of child development. Cambridge, England: Cambridge University Press.

Jacques, S., Zelazo, P. D., Kirkham, N. Z., \& Semcesen, T. K. (1999). Rule selection versus rule execution in preschoolers: An error-detection approach. Developmental Psychology, 35, 770-780.

Kirkham, N. Z., Cruess, L., \& Diamond, A. (2003). Helping children apply their knowledge to their behavior on a dimension-switching task. Developmental Science, 6, 449-467.

Liss, M., Fein, D., Allen, D., Dunn, M., Feinstein, C., Morris, R., et al. (2001). Executive functioning in high-functioning children with autism. Journal of Child Psychology and Psychiatry, 42, 261-270.

Luria, A. R., \& Homskaya, E. D. (1964). Disturbance in the regulative role of speech with frontal lobe lesions. In J. M. Warren \& K. Akert (Eds.), The frontal granular cortex and behavior (pp. 353-371). New York: McGraw-Hill.

Mayr, U., \& Keele, S. (2000). Changing internal constraints on action: The role of backward inhibition. Journal of Experimental Psychology: General, 129, 4-26.

Mischel, H. N., \& Mischel, W. (1983). The development of children's knowledge of self-control strategies. Child Development, 54, 603-619.

Munakata, Y. (2001). Graded representations in behavioral dissociations. Trends in Cognitive Sciences, $5,309-315$.

Munakata, Y., \& Yerys, B. E. (2001). All together now: When dissociations between action and knowledge disappear. Psychological Science, 12, 335-337.

Perner, J., \& Lang, B. (2002). What causes 3-year olds' difficulty on the dimensional change card sorting task? Infant \& Child Development, 11, 93-105.

Piaget, J. (1954). The construction of reality in the child. New York: Basic Books.

Roberts, A. C., De Salvia, M. A., Wilkinson, L. S., Collins, P., Muir, J. L., Everitt, B. J., et al. (1994). 6-Hydroxydopamine lesions of the prefrontal cortex in monkeys enhance performance on an analog of the Wisconsin card sort test: Possible interactions with subcortical dopamine. Journal of Neuroscience, 14, 2531-2544.

Rogers, R. D., Andrews, T. C., Grasby, P. M., Brooks, D. J., \& Robbins, T. W. (2000). Contrasting cortical and subcortical activations produced by attentional-set shifting and reversal learning in humans. Journal of Cognitive Neuroscience, 12, 142-162.

Russell, J., Mauthner, N., Sharpe, S., \& Tidswell, T. (1991). The windows task as a measure of strategic deception in preschoolers and autistic subjects. British Journal of Developmental Psychology, 9, 331-349.

Shallice, T. (1988). From neuropsychology to mental structure. Cambridge, England: Cambridge University Press.

Siegel, S. (1956). Nonparametric statistics for the behavioral sciences. New York: McGraw-Hill.

Stroop, J. R. (1935). Studies of interference in serial verbal reactions. Journal of Experimental Psychology, 18, 643-662.

Thompson, R. F. (1990). Neural mechanisms of classical conditioning in mammals. Philosophical Transactions of the Royal Society, B, 329, 161-170.

Towse, J. N., Redbond, J., Houston-Price, C. M. T., \& Cook, S. (2000). Understanding the dimensional change card sort: Perspectives from task success and failure. Cognitive Development, 15 , 347-365.

Zaitchik, D. (1991). Is only seeing really believing? Sources of the true belief in the false belief task. Cognitive Development, 6, 91-103. 
Zelazo, P. D., \& Frye, D. (1997). Cognitive complexity and control: A theory of the development of deliberate reasoning and intentional action. In M. Stamenov (Ed.), Language structure, discourse and the access to consciousness (pp. 113-153). Philadelphia: Benjamins.

Zelazo, P. D., \& Frye, D. (1998). Cognitive complexity and control: II. The development of executive function in childhood. Current Directions in Psychological Science, 7, 121-126.

Zelazo, P. D., Frye, D., \& Rapus, T. (1996). An age-related dissociation between knowing rules and using them. Cognitive Development, 11, 37-63.

Zelazo, P. D., \& Jacques, S. (1996). Children's rule-use: Representation, reflection and control. Annals of Child Development, 12, 119-176.

Zelazo, P. D., Reznick, J. S., \& Pinon, D. E. (1995). Response control and the execution of verbal rules. Developmental Psychology, 31, 508-517. 